\title{
Erratum to: 3rd International Symposium of Space Optical Instruments and Applications
}

\author{
H. Paul Urbach and Guangjun Zhang
}

\section{Erratum to:}

H.P. Urbach and G. Zhang (eds.), 3rd International Symposium of Space Optical Instruments and Applications, Springer Proceedings in Physics 192, DOI 10.1007/978-3-319-49184-4

The original version of the book was inadvertently published with spell errors in the authors' names. "Xuhie Huang" in Chapter "Optical Design of an Aperture-Divided MWIR Imaging Polarimeter" has to be changed to "Xujie Huang" and "Qiao-yun Fa" in Chapter "In-Orbit Calibration Method for Sun Sensor Based on Sun Ephemeris and Star Sensor" has to be changed to "Qiao-yun Fan". The erratum book has been updated with these changes. 